\title{
Trait anxiety predicts disease-specific health status in early-stage breast cancer patients
}

\author{
Lotje Van Esch · Jan A. Roukema • \\ Alida F. W. Van der Steeg $\cdot$ Jolanda De Vries
}

Accepted: 14 December 2010/Published online: 4 January 2011

(C) The Author(s) 2011. This article is published with open access at Springerlink.com

\begin{abstract}
Purpose The objectives of this study were to examine the differences in health status (HS) of women with breast cancer (BC) at different moments in time, and between women scoring high and not high on trait anxiety, and to identify possible predictors of HS 6 and 12 months after surgery.

Methods Patients $(N=223)$ completed a trait anxiety questionnaire before diagnosis. Women who received a diagnosis of $\mathrm{BC}$ completed a $\mathrm{BC}$-specific $\mathrm{HS}$ questionnaire 1, 3, 6 and 12 months after surgery. ANCOVA for repeated
\end{abstract}

L. Van Esch · J. A. Roukema - A. F. W. Van der Steeg .

J. De Vries

CoRPS, Center of Research on Psychology in Somatic Diseases,

P.O. Box 90153, 5000 LE Tilburg, The Netherlands

L. Van Esch · J. A. Roukema - A. F. W. Van der Steeg .

J. De Vries $(\triangle)$

CoRPS, Department of Medical Psychology, Tilburg University,

P.O. Box 90153, 5000 LE Tilburg, The Netherlands

e-mail: J.devries@uvt.nl; jvries@elisabeth.nl

URL: www.tilburguniversity.nl/corps

J. A. Roukema

Department of Surgery, St. Elisabeth Hospital,

P.O. Box 90151, 5000 LC Tilburg, The Netherlands

A. F. W. Van der Steeg

Pediatric Surgical Center of Amsterdam, Emma Children's

Hospital AMC, Amsterdam, The Netherlands

A. F. W. Van der Steeg

VU Medical Center, P.O. Box 22660, 1100 DD Amsterdam,

The Netherlands

J. De Vries

Department of Medical Psychology, St. Elisabeth Hospital,

P.O. Box 90151, 5000 LC Tilburg, The Netherlands measures and multiple regression analysis were used in the analyses.

Results Women scoring high on trait anxiety had significant $(P<.005)$ lower Body image, worse Future perspective and Sexual functioning, and more Side-effects than women who did not score high on trait anxiety. At 6 and 12 months after surgery, the same aspects of HS were predicted by higher trait anxiety scores.

Conclusions Higher scores on trait anxiety resulted in worse scores on four HS domains, indicating that there should be more attention for this group of patients, even before treatment starts.

Keywords Oncology - Breast cancer - Health status . Trait anxiety $\cdot$ Treatment

\section{Introduction}

Breast cancer is the most widespread form of cancer in Europe [1]. Since incidence rates are still increasing but mortality is decreasing [1,2], more and more women are survivors of breast cancer. For these women, health status (HS), defined as "well-being in terms of physical, mental, and social condition" [3], is an important outcome, next to their medical condition. HS has been examined in different studies with various measures, for instance among longterm survivors ( $>5$ years) of breast cancer [4]. In these women, overall HS was fairly good. The most frequently reported complaints were sexual problems and arm problems [4]. When HS was measured within 2 years after diagnosis, DiSipio et al. [5] found that younger breast cancer survivors $(<50$ years) 1 year after diagnosis had a HS comparable with the normal population. Women of 50 years and older already had a HS comparable with the 
normal population 6 months after diagnosis. Inconsistent with these results, other studies noted that 1 year after surgery, cognitive and social functioning in breast cancer survivors were impaired compared with the normal population [6], and the HS of younger women was more impaired than that of older women $[7,8]$. There are recent studies that indicate that HS is also affected by socioeconomic status and comorbidity [9, 10].

A factor that is associated with the level of HS in breast cancer patients, although not studied frequently, is trait anxiety, i.e. the disposition to experience anxiety in threatening situations. Schreier et al. [11] for example, found that trait anxiety correlates negatively with HS scores in breast cancer patients. Trait anxiety also predicted higher levels of distress in breast cancer patients [12], with higher levels of distress being directly related to a decrease in HS [13]. A recent study in patients with rectal cancer showed that higher trait anxiety levels predicted worse HS [14].

In this longitudinal, prospective study, trait anxiety was measured in patients before they received a diagnosis. Subsequently, HS was measured at different moments in time after diagnosis and treatment. This makes it possible to look at the relation between trait anxiety and the different aspects of HS, as well as looking at the course of HS in time.

The goals of this study were to examine (1) whether women who score high on trait anxiety have a different HS across time compared with women who do not score high on trait anxiety, and (2) which factors predict HS 6 months and 1 year after surgery. The expectation was that women who scored high on trait anxiety had a worse HS than women who did not score high on trait anxiety. Furthermore, trait anxiety was expected to predict HS at 6 and 12 months after surgery.

\section{Methods and patients}

\section{Patients}

This study was part of a larger study, concerning the relationship between personality, surgical treatment and quality of life in women with early-stage breast cancer. In this larger study, all women visiting the outpatient clinic of the St Elisabeth Hospital in Tilburg (from January 2002 until June 2007), the Maasland Hospital in Sittard (from June 2004 until March 2006), and the Jeroen Bosch Hospital in Den Bosch (from January 2006 until June 2007), The Netherlands, were asked to participate. They were referred by their general practitioner with either a palpable lump in the breast or an abnormality on the screening mammography and a negative medical history for breast disease. At their first appointment with a specialized mamma-care nurse (a nurse who is specialized in the care for breast cancer patients), they were informed about the study and when women agreed to participate, they completed an informed consent and a set of questionnaires (Time 1). This was before they knew the diagnosis of the breast problem they visited the outpatient clinic for. Of the 799 women who visited one of the outpatient clinics with a problem of the breast, $286(35.8 \%)$ were diagnosed with early-stage breast cancer. The other 513 (64.2\%) patients appeared to have a benign breast problem. Of the 286 women with breast cancer, $223(78 \%)$ completed the first set of questionnaires, including a questionnaire about trait anxiety. Patients with breast cancer included in this study were in the situation that they had a choice in their surgical treatment: they could choose either for breast conserving therapy (BCT) or for mastectomy (MTC). The breast cancer patients received additional questionnaires at 1 (Time 2), 3 (Time 3), 6 (Time 4) and 12 months (Time 5) after surgical treatment (see Fig. 1). Exclusion criteria for the current study were not being able to speak and/or read Dutch, having cognitive problems, a medical history with breast disease, advanced-stage breast cancer or a benign breast problem. This study was approved by the Medical Ethical Committee (ccMo NL15659.008.06).

\section{Questionnaires}

HS was measured at Time 2, Time 3, Time 4, and Time 5 using the validated disease-specific European Organisation for Research and Treatment of Cancer Quality of Life Questionnaire Breast Cancer module (EORTC QLQBR23) [15, 16]. This 23-item questionnaire is developed for breast cancer patients at different stages of their disease and with varying treatment modalities. The EORTC QLQBR23 measures the functional scales Body image (4 items), Sexual functioning (2 items), Sexual enjoyment (1 item;

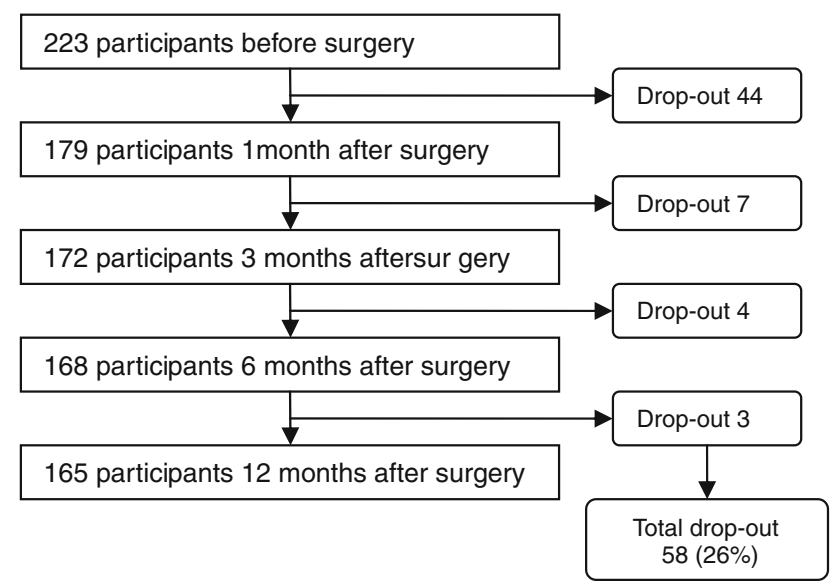

Fig. 1 Flowchart of the study 
only to be completed when patient is sexually active), and Future perspective ( 1 item; "Were you worried about your health in the future?") and the disease symptom scales Systemic therapy side-effects (7 items), Breast symptoms (4 items), Arm symptoms ( 3 items), and Upset by hair loss (1 item; only to be completed if patient had hair loss). Answers vary from 1 (not at all) to 4 (very much). Higher scores on the functional scales represent a higher level of functioning, while higher scores on the symptom scales represent more symptomatology.

Trait anxiety was measured at Time 1 with the trait anxiety subscale of the State-Trait Anxiety Inventory (STAI) at Time 1 [17, 18]. The STAI measures trait anxiety with 20 items. Answers range from 1 (almost never) to 4 (almost always). It is a frequently used questionnaire with good psychometric properties [18]. For some analyses, the scores on trait anxiety were dichotomized in high or not high. A cut-off score of 44 or higher was used to determine high trait anxiety [18]. The definition of this cut-off score was based on normative data for women between 40 and 59 years [19] and was construed using the mean score (34.2) plus $1 \mathrm{SD}$ (9.87).

Demographic variables included in the study were age and marital status. Level of education and work status were assessed to use as an approximation of socio-economic status.

\section{Medical records}

The following information was retrieved from patients' medical files: the disease stage at diagnosis [20], type of operation, and adjuvant treatment (chemotherapy, radiotherapy, hormone treatment).

\section{Statistical procedure}

For baseline measures, Chi-square (discrete variables) and independent samples $T$ tests (continuous variables) were used to compare participants and non-participants, and the trait anxiety groups (dichotomized in high or not high) with regard to age, partner, children, education, work, disease stage at diagnosis, level of trait anxiety, surgical treatment, and adjuvant treatment. ANCOVA for repeated measures was used to examine differences in HS scores between measurements in time and between patients with different trait anxiety scores (dichotomized in high or not high). Additionally, univariate regression analysis was used to select factors for the multivariate linear regression analyses (MRA). The dependent variables in the univariate regression were Body image, Sexual functioning, Future perspective, Systemic therapy side-effects, Breast symptoms and Arm symptoms at T4 and T5. Independent variables were age, partner yes/no, children yes/no, education, work yes/no, level of trait anxiety (continuous total score), screening referral yes/no, disease stage at diagnosis, surgical treatment, adjuvant therapy yes/no, chemotherapy yes/no, radiotherapy yes/no, and hormone therapy yes/no. The significant independent variables of the univariate regression analysis were imported in the backward MRA that was performed to identify those factors that best predicted patients' scores on the EORTC QLQ-BR23 at Time 4 and Time 5. All analyses were performed with the Statistical Package for Social Sciences (SPSS) version 14.0.

\section{Results}

Of 286 eligible women with breast cancer, 223 (78\%) participated in the study. There were no differences in age, disease stage, type of operation, and receiving adjuvant therapy between participants and non-participants. Demographic and medical information of participants is summarized in Table 1.

About $40 \%$ was diagnosed with disease stage I. Regarding surgical treatment, 107 women had BCT and 114 women received MTC. Women with BCT and MTC significantly differed on receiving adjuvant therapy and disease stage; i.e. BCT patients more often had adjuvant therapy $(P<.001)$ and were in a lower disease stage at diagnosis $(P=.002)$. Women scoring high on trait anxiety (37\%) had a lower education level $(P=.005)$ and were older $(P=.041)$ than women not scoring high on trait anxiety. There were no differences concerning other demographic and medical factors. Disease stage, receiving adjuvant therapy, type of surgery, educational level, and age were included as covariates in the subsequent analyses. During the study, 58 women (26\%) dropped out within 12 months after surgery. Reasons for drop-out were 'not interested', 'do not want to be confronted with the past,' or 'too much work' (Fig. 1). Participants and drop-outs only differed on age $(P=.008)$, with drop-outs being older than participants.

In the MRA, the total score on trait anxiety was included following the goal of the study. Age, disease stage, receiving adjuvant therapy, type of surgery, and educational level were included because these factors differed between groups. In addition, significant factors of the univariate analyses were included, namely chemotherapy and screening referral for Body image, marital status for Sexual functioning, chemotherapy and hormone therapy for Systemic therapy side-effects, and children, radiotherapy, and screening referral for Breast symptoms (see Table 2).

Functional scales

The high trait anxiety group had a lower Body image than the no-high group $(P=.003)$. The covariate type of surgery was also significant. The BCT group had a higher 
Table 1 Demographic and medical information of the breast cancer patients $(N=223)$

* Screening referral Women referred by the national breast cancer screening program

\begin{tabular}{lllr}
\hline Demographic information & Medical information \\
\hline Age & & Screening referral* \\
Mean \pm SD & $58.7 \pm 9.4$ & Yes & $151(67.7 \%)$ \\
Partner & & No & $72(32.3 \%)$ \\
Yes & $179(80.2 \%)$ & Disease stage \\
No & $37(16.6 \%)$ & Stage 0 & $24(10.8 \%)$ \\
Missing & $7(3.1 \%)$ & Stage I & $93(41.7 \%)$ \\
Children & & Stage IIa & $70(31.4 \%)$ \\
Yes & $191(85.7 \%)$ & Stage IIb & $35(15.7 \%)$ \\
No & $29(13.0 \%)$ & Indefinable & $1(0.4 \%)$ \\
Missing & $3(1.3 \%)$ & Type of operation & $107(48.0 \%)$ \\
Educational level & & Breast conserving therapy & $114(51.1 \%)$ \\
Low & $85(38.1 \%)$ & Mastectomy & $2(0.9 \%)$ \\
Middle & $95(42.6 \%)$ & No operation & $60(26.9 \%)$ \\
High & $37(16.6 \%)$ & Adjuvant therapy & $117(52.2 \%)$ \\
Missing & $6(2.7 \%)$ & Received chemotherapy & $87(39.0 \%)$ \\
Work status & & Received radiotherapy & \\
Employed & $85(38.1 \%)$ & Received hormone treatment & \\
Unemployed & $135(60.5 \%)$ & & \\
Missing & $3(1.3 \%)$ & & \\
\hline & & & \\
& & &
\end{tabular}

Table 2 Outcomes of the univariate regression analyses of demographic and medical factors on the EORTC QLQ-BR23 domains: $P$ values

\begin{tabular}{|c|c|c|c|c|c|c|c|c|c|c|c|c|}
\hline & BI T4 & FP T4 & SF T4 & SE T4 & BS T4 & AS T4 & BI T5 & FP T5 & SF T5 & SE T5 & BS T5 & AS T5 \\
\hline Age & .002 & & & & & & .025 & & .001 & & & \\
\hline Screening referral & .014 & & & & .042 & & & & & & & \\
\hline STAI total score & .006 & $<.001$ & $<.001$ & .004 & .034 & & .020 & $<.001$ & .011 & .008 & .022 & \\
\hline Adjuvant treatment & & & & & & & & & & & .016 & \\
\hline Chemotherapy & .001 & & & $<.001$ & & & .038 & & & & & \\
\hline Radiotherapy & & & & & .003 & & & & & & $<.001$ & \\
\hline Hormonal treatment & & & & .029 & & & & & & & & \\
\hline Type of surgery & .001 & & & & .007 & & .005 & & & & $<.001$ & \\
\hline Disease stage & .045 & & & .003 & & & & & & & & \\
\hline Educational level & & & & & .017 & .018 & & & & & .012 & \\
\hline Children & & & & & .017 & & & & & & & \\
\hline Work & & & & & & & & & & & & \\
\hline Partner & & & .007 & & & & & & $<.001$ & & & \\
\hline
\end{tabular}

$B I$ Body image, $F P$ future perspective, $S F$ sexual functioning, $S E$ systemic therapy side-effects, $B S$ breast symptoms, $A S$ arm symptoms, $T 4$ measurement 6 months after surgical treatment, T5 measurement 12 months after surgical treatment

Note: Only significant results are shown

Body image than the MTC group at all measurements $(P=.006)$. More positive Body image scores at Time 4 and Time 5 was predicted by lower scores on trait anxiety, BCT, and older age. Receiving chemotherapy predicted a more negative Body image, but only at Time 4 (Table 3 ).

Women who did not score high on trait anxiety had a more positive Future perspective than women who did score high on trait anxiety $(P<.001)$ (see Fig. 2$)$. The latter group had a more unusual course of Future perspective. Between Time 3 and Time 4 their Future perspective dropped seriously $(P<.001)$. At Time 5 , their Future perspective had increased to approximately their Time 2 level. This pattern is reflected in the interaction effect of time and trait anxiety $(P<.001)$.

More positive Future perspective at Time 4 was predicted by lower scores on trait anxiety and older age. Trait 
Table 3 Predictors of the functional scales at 6 and 12 months after surgery

\begin{tabular}{|c|c|c|c|c|c|c|}
\hline & \multicolumn{3}{|c|}{6 months after surgery } & \multicolumn{3}{|c|}{12 months after surgery } \\
\hline & $\beta$ & $P$ & Tot. adj. $R^{2}$ & $\beta$ & $P$ & Tot. adj. $R^{2}$ \\
\hline \multicolumn{7}{|l|}{ Body image } \\
\hline Trait anxiety & -.311 & $<.001$ & & -.257 & .002 & \\
\hline Type of surgery & -.239 & .002 & & -.266 & .001 & \\
\hline Age & .259 & .001 & & .256 & .002 & \\
\hline Chemotherapy & -.173 & .031 & $23.5 \%$ & & & $15.7 \%$ \\
\hline \multicolumn{7}{|l|}{ Future perspective } \\
\hline Trait anxiety & -.497 & $<.001$ & & -.335 & $<.001$ & \\
\hline Age & .166 & .025 & $25.0 \%$ & & & $10.5 \%$ \\
\hline \multicolumn{7}{|l|}{ Sexual functioning } \\
\hline Trait anxiety & -.330 & $<.001$ & & -.232 & .007 & \\
\hline Partner & .192 & .023 & & .277 & .002 & \\
\hline Age & & & $13.2 \%$ & -.212 & .015 & $17.5 \%$ \\
\hline
\end{tabular}

Future Perspective

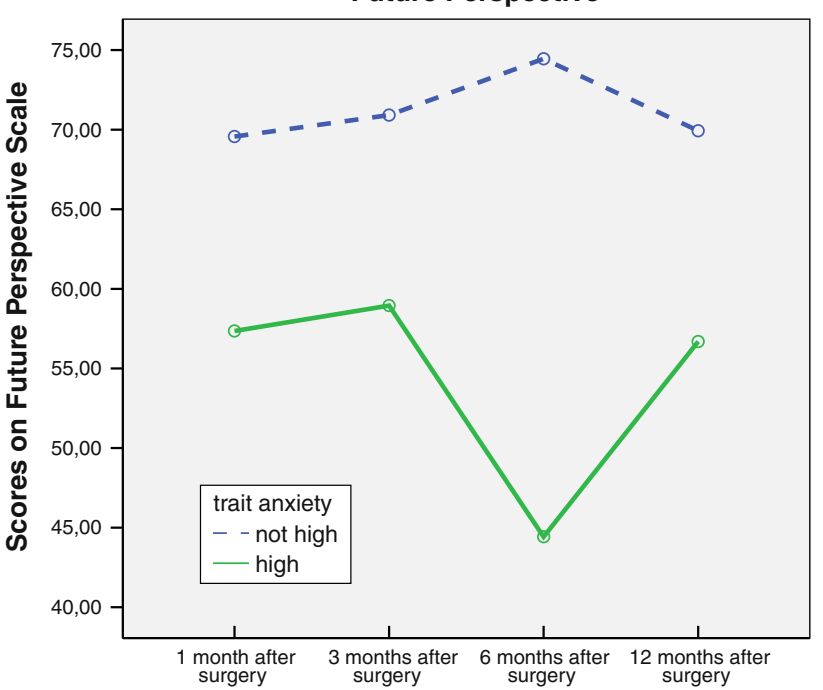

Fig. 2 Scores on the Future Perspective scale of the EORTC QLQBR23 for women scoring high and not high on trait anxiety

anxiety was the only predictor of more positive Future perspective at Time 5 (Table 3), i.e., lower scores on trait anxiety predicted higher Future perspective.

The women with a high score on trait anxiety reported lower Sexual functioning than the other women $(P=$ $.002)$. There was an interaction effect between time and chemotherapy, showing that the course of Sexual functioning for women with or without chemotherapy differed across time $(P=.045)$. Two different factors predicted better Sexual functioning at Time 4 and Time 5, namely lower scores on trait anxiety and having a partner. Younger age was a predictor of a higher level of Sexual functioning at Time 5 (Table 3).

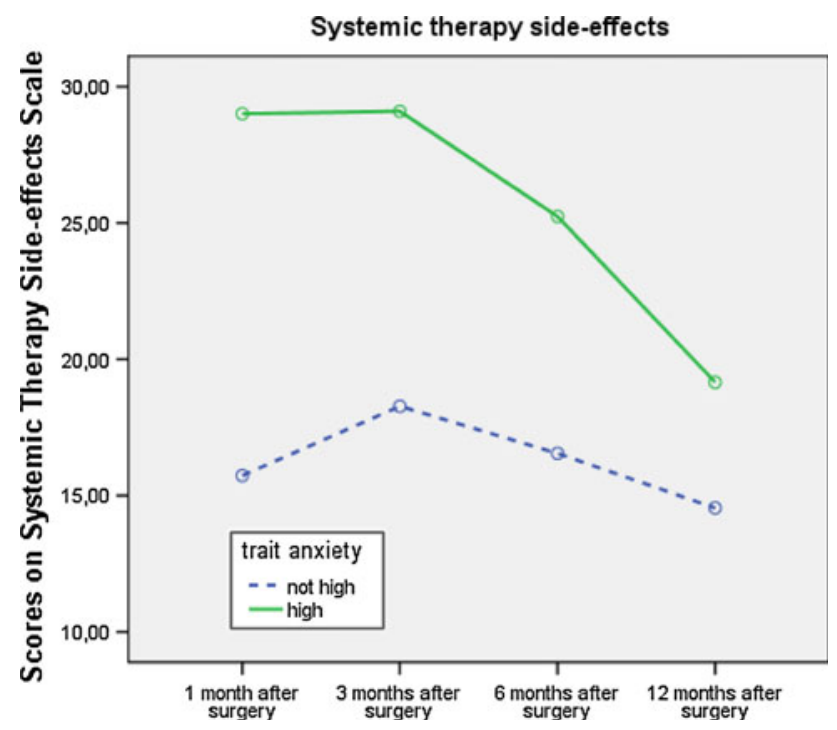

Fig. 3 Scores on the Systemic Therapy Side-effects scale of the EORTC QLQ-BR23 for women scoring high and not high on trait anxiety

\section{Symptom scales}

Women high on trait anxiety reported more Side-effects than the other women $(P=.001)$ (Fig. 3). There was an interaction effect between time and chemotherapy $(P=.012)$ reflecting that women without chemotherapy have a constant level of Side-effects across time and women with chemotherapy experienced a major increase in Side-effects 3 months after surgery. Side-effects decreased toward 12 months after surgery, but the level stayed above that of women without chemotherapy. Receiving chemotherapy and having a higher score on trait anxiety were significant predictors of more Side-effects at Time 4. At 
Table 4 Predictors of the symptoms scales at 6 and 12 months after surgery

\begin{tabular}{|c|c|c|c|c|c|c|}
\hline & \multicolumn{3}{|c|}{6 months after surgery } & \multicolumn{3}{|c|}{12 months after surgery } \\
\hline & $\beta$ & $P$ & Tot. adj. $R^{2}$ & $\beta$ & $P$ & Tot. adj. $R^{2}$ \\
\hline \multicolumn{7}{|l|}{ Side-effects } \\
\hline Chemotherapy & .340 & $<.001$ & & & & \\
\hline Trait anxiety & .299 & $<.001$ & $16.9 \%$ & .249 & .005 & $5.3 \%$ \\
\hline \multicolumn{7}{|l|}{ Breast symptoms } \\
\hline Radiotherapy & .251 & .002 & & & & \\
\hline Children & -.233 & .004 & & & & \\
\hline Educational level & -.223 & .006 & & -.224 & .008 & \\
\hline Type of surgery & & & $14.3 \%$ & -.378 & $<.001$ & $17.3 \%$ \\
\hline \multicolumn{7}{|l|}{ Arm symptoms } \\
\hline Educational level & -.204 & .016 & $3.5 \%$ & -.177 & .048 & $2.3 \%$ \\
\hline
\end{tabular}

Time 5, only higher scores on trait anxiety significantly predicted more Side-effects (see Table 4).

Trait anxiety did not interfere with Breast symptoms, but type of surgery did. BCT resulted in significantly $(P=.007)$ more Breast symptoms than MTC. More Breast symptoms at Time 4 were predicted by receiving radiotherapy, a lower educational level and not having children. Educational level remained a predictor at Time 5, and receiving BCT predicted more Breast symptoms (Table 4).

Arm symptoms differed between women in different disease stages $(P=.015)$. A higher disease stage resulted in more Arm symptoms. Worse scores on Arm symptoms at Time 4 and Time 5 were predicted by lower educational level (see Table 4).

\section{Discussion}

In this study, we examined the relationship between trait anxiety and HS, taking into account multiple demographic, personality, and medical factors. HS contains six domains in this study, and four of those domains, namely Body image, Future perspective, Sexual functioning, and Sideeffects were negatively affected by trait anxiety.

\section{Trait anxiety}

One of the goals of this study was to examine whether there were differences in HS between women who scored high on trait anxiety and women who did not score high on trait anxiety. Based on earlier studies [11, 12, 21], we expected that women with a high score on trait anxiety would have lower scores on HS. Indeed trait anxiety seemed to play an important role in the HS of women with breast cancer, especially with regard to Body image, Future perspective, Sexual functioning, and Side-effects. On these scales of the EORTC QLQ-BR23, women who scored high on trait anxiety had significant worse outcome than women who did not score high on trait anxiety, even 12 months after surgical treatment. A remarkable result was, for example, the course of Future perspective of women with high scores on trait anxiety (see Fig. 2). There is a significant decrease in their Future perspective 6 months after surgery took place. An explanation could be that when treatment has ended, patients are uncertain about how to pick up their lives.

Furthermore, trait anxiety was the only factor that significantly and negatively predicted Body image, Future perspective and Sexual functioning at 6 and 12 months after surgery, implying that other factors such as disease stage and treatment have less impact on HS than this personality trait. Comparable results were found in three other studies, even though in these studies (HR) QoL [11, 21] and psychological stress [12] were used as outcome measures.

Moreover, higher trait anxiety predicted more Systemic therapy side-effects at 6 and 12 months after surgery (see Fig. 3). This could be due to the fact that patients scoring high on trait anxiety believe that they will experience more side-effects of their systemic therapy, as mentioned in the study of Cameron and colleagues [22]. There was no relation between trait anxiety and Arm and Breast symptoms.

\section{Demographic factors}

Another factor besides trait anxiety that predicted more than one domain of HS was age. Older age predicts a better Body image at 6 and 12 months after surgery and a more positive perspective on the future at 6 months after surgery. Multiple explanations could be considered here. One possibility is that younger women more often have an advanced stage of breast cancer, or a more aggressive tumor. In these cases, treatment can be more invasive and mutilating and more often includes chemotherapy, which is 
also a predictor for worse $\mathrm{HS}$ [5, 7]. Another explanation could be that a diagnosis of $\mathrm{BC}$ and the subsequent treatment possibly disrupts the course of life more in younger women than in older women [23, 24].

Contrary to the previous result where older age was related to more positive outcomes, better Sexual functioning at 12 months after surgery was predicted by younger age. Presumably, this has a relation with menopause and menopausal symptoms. Most of the older women have past their menopause, but in younger women, only those who had systemic therapy might have menopausal symptoms which can lead to decreased sexual enjoyment [7]. This relation between age and Sexual functioning was also found in other studies that used the EORTC QLQ-BR23 [25, 26].

Other demographic factors with an influence on HS were educational level, having children, and having a partner. A higher educational level predicted less Breast and Arm symptoms at 6 months after surgery, and less Breast symptoms at 12 months after surgery. Earlier research revealed the same relation between education and Breast and Arm symptoms [27]. A possible reason for this could be that higher educated women are more likely to learn about how to take care of their wounds and how to exercise and use their arm. Having children predicted less Breast symptoms at 6 months after surgery, and having a partner predicted better Sexual functioning at 6 and 12 months after surgery.

\section{Disease-specific factors: treatment}

Type of surgery had a significant relation with Body image: women that had BCT had a better Body image than women that had MTC, during the 12 months after surgery. This outcome is also supported by multiple studies [28-30]. A more unexpected result was that women with BCT reported much more Breast symptoms than women that had MTC. BCT also significantly predicted more Breast symptoms at Time 5. Since MTC is medically more invasive than BCT, it would be obvious to expect that MTC causes more Breast symptoms. The current outcome could possibly be attributed to treatment features: in BCT radiotherapy is part of regular treatment, while women that have MTC almost never receive radiotherapy. Radiotherapy can therefore not be seen apart from type of surgical treatment. From the regression analysis, we also learned that not receiving radiotherapy was a predictor for less Breast symptoms. These facts together can obviously explain why women with BCT have more Breast symptoms: radiotherapy can cause irritation of the skin and wounds heal slower [31]. Another possible explanation is a linguistic one: in the questionnaire, women are asked about complaints in the area of their affected breast. Perhaps, women that had an
MTC reason that they do not have a breast anymore (for MTC removes the breast in total), and they do not report possible complaints as complaints of their breast. In the study of King et al. [27], there was no significant difference reported in Breast symptoms between BCT and MTC, and apart from that study there is no specific literature about this topic, so more research is needed to clarify this result.

Besides type of surgery, there were other treatment factors that predicted HS. Receiving chemotherapy predicted more Side-effects and worse Body image at Time 4. Radiotherapy predicted worse Future perspective and more Breast symptoms at Time 4 . These results are in line with a recent study on adjuvant therapy and HR-QoL [32]. In contrast to what Buijs et al. [33] found, there was a relation between hormone therapy and Arm symptoms: receiving hormone therapy predicted less Arm symptoms at Time 5. This could have a relation with other factors, like radiotherapy, but more research needs to be done about this topic.

\section{Limitations of this study}

In this study, only women with early-stage breast cancer were included (see Table 1). An advantage is the homogeneity of the data, but the disadvantage is that it is not possible to generalize the conclusions to other patient groups, such as late-stage or recurrent breast cancer patients. Furthermore, there was a selection effect on two items of the EORTC QLQ-BR23: the items about 'Upset by hair loss' and 'Sexual enjoyment' only had to be answered if women actually did have hair loss or were sexually active, respectively. Due to these criteria, not enough patients answered the questions to evaluate them in this study. To counter this problem and other potential power problems, more women need to be included in future research.

In this study, radiotherapy was introduced as an independent variable. We realize that there is an overlap between radiotherapy and type of surgery, i.e. usually women who receive BCT also get radiotherapy. Likewise, radiotherapy is usually not a part of MTC treatment. However, because in this study there were 10 women ( $9.3 \%$ of BCT) who received BCT but not radiotherapy, and 20 women (17.5\% of MTC) who received MTC and radiotherapy, we decided to maintain radiotherapy as an independent variable.

In conclusion, trait anxiety is strongly associated with the HS of breast cancer patients in the first year after surgery. This insight into the personality of patients could be a first indication of better monitoring patients that have a higher risk of having a worse HS after diagnosis and treatment of their disease. Since HS is an important endpoint of treatment in breast cancer patients nowadays, psychosocial support should be deployed to guide these 
high-risk patients through the process of their disease and possibly thereafter.

Furthermore, it should not be accepted unquestioningly that women who had BCT do better than women with MTC. The results from this study pointed out that even though BCT is, medically spoken, a less invasive treatment than MTC, BCT patients can have serious Breast symptoms. There should therefore be close attention for medical and psychosocial symptoms in women with MTC and BCT.

The results of this study also have clinical implications. Based on the present results, women scoring high on anxiety are prone to experiencing more side-effects. Psychological counseling aimed at these women could probably reduce side-effects. However, in order to provide this treatment, high anxious women should be identified using short, validated easy-to-administer trait anxiety questionnaires, such as the STAI [17, 18].

Open Access This article is distributed under the terms of the Creative Commons Attribution Noncommercial License which permits any noncommercial use, distribution, and reproduction in any medium, provided the original author(s) and source are credited.

\section{References}

1. Ferlay, J., Autier, P., Boniol, M., Heanue, M., Colombet, M., \& Boyle, P. (2007). Estimates of the cancer incidence and mortality in Europe in 2006. Annals of Oncology, 18(3), 581-592.

2. Jatoi, I., \& Miller, A. B. (2003). Why is breast-cancer mortality declining? Lancet Oncol, 4(4), 251-254.

3. An international glossary for general/family practice. WONCA Classification Committee. (1995). Family Practice, 12(3), 341-369.

4. Mols, F., Vingerhoets, A. J., Coebergh, J. W., \& Van de PollFranse, L. V. (2005). Quality of life among long-term breast cancer survivors: a systematic review. European Journal of Cancer, 41(17), 2613-2619.

5. Disipio, T., Hayes, S., Newman, B., \& Janda, M. (2008). Healthrelated quality of life 18 months after breast cancer: comparison with the general population of Queensland, Australia. Supportive Care in Cancer, 16(10), 1141-1150.

6. Ahn, S. H., Park, B. W., Noh, D. Y., Nam, S. J., Lee, E. S., Lee, M. K., et al. (2007). Health-related quality of life in disease-free survivors of breast cancer with the general population. Annals of Oncology, 18(1), 173-182.

7. Hopwood, P., Haviland, J., Mills, J., Sumo, G., \& Bliss, J. M. (2007). The impact of age and clinical factors on quality of life in early breast cancer: an analysis of 2,208 women recruited to the UK START trial (standardisation of breast radiotherapy trial). Breast, 16(3), 241-251.

8. Engel, J., Kerr, J., Schlesinger-Raab, A., Sauer, H., \& Holzel, D. (2004). Quality of life following breast-conserving therapy or mastectomy: results of a 5-year prospective study. Breast Journal, 10(3), 223-231.

9. Shin, H. W., Noh, D. Y., Lee, E. S., Nam, S. J., Park, B. W., Ahn, S. H., et al. (2009). Correlates of existential well-being and their association with health-related quality of life in breast cancer survivors compared with the general population. Breast Cancer Research and Treatment, 118(1), 139-150.
10. Zebrack, B. J., Yi, J., Petersen, L., \& Ganz, P. A. (2008). The impact of cancer and quality of life for long-term survivors. Psychooncology, 17(9), 891-900.

11. Schreier, A. M., \& Williams, S. A. (2004). Anxiety and quality of life of women who receive radiation or chemotherapy for breast cancer. Oncology Nursing Forum, 31(1), 127-130.

12. Bleiker, E. M., Pouwer, F., Van der Ploeg, H. M., Leer, J. W., \& Ader, H. J. (2000). Psychological distress two years after diagnosis of breast cancer: frequency and prediction. Patient Education and Counseling, 40(3), 209-217.

13. Hevey, D., McGee, H. M., \& Horgan, J. (2007). Relationship of initial level of distress to changes in health-related quality of life during cardiac rehabilitation or usual care. Psychosomatic Medicine, 69(8), 793-797.

14. Ristvedt, S. L., \& Trinkaus, K. M. (2009). Trait anxiety as an independent predictor of poor health-related quality of life and post-traumatic stress symptoms in rectal cancer. British Journal of Health Psychology, 14(Pt 4), 701-715.

15. Sprangers, M. A., Groenvold, M., Arraras, J. I., Franklin, J., Te Velde, A., Muller, M., et al. (1996). The European organization for research and treatment of cancer breast cancer-specific quality-of-life questionnaire module: first results from a three-country field study. Journal of Clinical Oncology, 14(10), 2756-2768.

16. Montazeri, A., Harirchi, I., Vahdani, M., Khaleghi, F., Jarvandi, S., Ebrahimi, M., et al. (2000). The EORTC breast cancer-specific quality of life questionnaire (EORTC QLQ-BR23): translation and validation study of the Iranian version. Quality of Life Research, 9(2), 177-184.

17. Spielberger, C. D., Gorsuch, R. L., \& Lushene, R. E. (1970). The State-Trait Anxiety Inventory manual. Palo Alto, CA: Consulting Psychologists Press.

18. Van der Ploeg, H. M., Defares, P. B., \& Spielberger, C. D. (1980). ZBV. A Dutch-language adaptation of the Spielberger State-Trait Anxiety Inventory. Lisse, The Netherlands: Swets\& Zeitlinger.

19. Spielberger, C., Gorsuch, R., Lushene, R., Vagg, P., \& Jacobs, G. (1983). Manual for the State-Trait Anxiety Inventory. Palo Alto, CA: Consulting Psychologists Press.

20. Greene, F. L., Page, D. L., Fleming, I. D., Fritz, A. G., Balch, C. M., Haller, D. G., et al. (2002). American joint committee on cancer staging manual (6th ed.). New York, NY: Springer.

21. Van der Steeg, A. F., De Vries, J., Van der Ent, F. W., \& Roukema, J. A. (2007). Personality predicts quality of life six months after the diagnosis and treatment of breast disease. Annals of Surgical Oncology, 14(2), 678-685.

22. Cameron, L. D., Leventhal, H., \& Love, R. R. (1998). Trait anxiety, symptom perceptions, and illness-related responses among women with breast cancer in remission during a tamoxifen clinical trial. Health Psychology, 17(5), 459-469.

23. Ganz, P. A., Greendale, G. A., Petersen, L., Kahn, B., \& Bower, J. E. (2003). Breast cancer in younger women: reproductive and late health effects of treatment. Journal of Clinical Oncology, 21(22), 4184-4193.

24. Hartl, K., Schennach, R., Muller, M., Engel, J., Reinecker, H., Sommer, H., et al. (2010). Quality of life, anxiety, and oncological factors: a follow-up study of breast cancer patients. Psychosomatics, 51(2), 112-123.

25. Browall, M. M., Ahlberg, K. M., Persson, L. O., Karlsson, P. O., \& Danielson, E. B. (2008). The impact of age on HHealthRelated Quality of Life (HRQoL) and symptoms among postmenopausal women with breast cancer receiving adjuvant chemotherapy. Acta Oncologica, 47(2), 207-215.

26. Fehlauer, F., Tribius, S., Mehnert, A., \& Rades, D. (2005). Health-related quality of life in long term breast cancer survivors treated with breast conserving therapy: impact of age at therapy. Breast Cancer Research and Treatment, 92(3), 217-222. 
27. King, M. T., Kenny, P., Shiell, A., Hall, J., \& Boyages, J. (2000). Quality of life three months and 1 year after first treatment for early stage breast cancer: influence of treatment and patient characteristics. Quality of Life Research, 9(7), 789-800.

28. De Haes, J. C., van Oostrom, M. A., \& Welvaart, K. (1986). The effect of radical and conserving surgery on the quality of life of early breast cancer patients. European Journal of Surgical Oncology, 12(4), 337-342.

29. Curran, D., Van Dongen, J. P., Aaronson, N. K., Kiebert, G., Fentiman, I. S., Mignolet, F., et al. (1998). Quality of life of early-stage breast cancer patients treated with radical mastectomy or breast-conserving procedures: results of EORTC Trial 10801. The European Organization for Research and Treatment of Cancer (EORTC), Breast Cancer Co-operative Group (BCCG). European Journal of Cancer, 34(3), 307-314.

30. Kenny, P., King, M. T., Shiell, A., Seymour, J., Hall, J., Langlands, A., et al. (2000). Early stage breast cancer: costs and quality of life 1 year after treatment by mastectomy or conservative surgery and radiation therapy. Breast, 9(1), 37-44.

31. Lilla, C., Ambrosone, C. B., Kropp, S., Helmbold, I., Schmezer, P., von Fournier, D., et al. (2007). Predictive factors for late normal tissue complications following radiotherapy for breast cancer. Breast Cancer Research and Treatment, 106(1), 143-150.

32. Browall, M., Ahlberg, K., Karlsson, P., Danielson, E., Persson, L. O., \& Gaston-Johansson, F. (2008). Health-related quality of life during adjuvant treatment for breast cancer among postmenopausal women. European Journal of Oncology Nursing, 12(3), 180-189.

33. Buijs, C., de Vries, E. G., Mourits, M. J., \& Willemse, P. H. (2008). The influence of endocrine treatments for breast cancer on health-related quality of life. Cancer Treatment Reviews, 34(7), 640-655. 